\title{
Value Oriented Cost-effectiveness Estimation of an Innovative Intermodal Loading Unit
}

\author{
S. Jursch, S. Bischoff, E. Hauck, P. Flachskampf, K. Henning and S. Jeschke
}

\begin{abstract}
The success of intermodal transport solutions compared to exclusive road transport significantly depends on the cost efficiency, the improvement of interoperability and the exploitation of a maximized cargo area. Intermodal transport provides the opportunity to benefit from the advantages of each of the individual transport modes integrated in a system that is both cost effective and sustainable. The development of an innovative intermodal loading unit (ILU), a seamless and customer-oriented transport concept, marks an important step towards enhancing competitiveness. The paper focuses on the method of the Value Oriented Cost-Effectiveness Estimation. This method had been developed by the Institute for Management Cybernetics at RWTH Aachen University and combines the classical analysis of investment in monetary terms with relevant non-monetary variables or the so-called soft factors in the profitability analysis. In this paper, a new approach of the Value Oriented Cost-Effectiveness Estimation is presented and applied in excerpts within the TelliBox project "Intelligent MegaSwapBoxes for Advanced Intermodal Freight Transport".
\end{abstract}

Index Terms-Effectiveness Estimation; TelliBox; Intermodality;Transport; Intermodal Loading Unit

\section{INTRODUCTION}

\section{A. Initial Situation}

In the last decades, intermodal freight transport was driven by the changing requirements of global supply chains. The improved integration and compatibility between modes provided the necessary scope for a sustainable transport system and the promotion of intermodality offered the opportunity to improve rail, inland waterways and short sea shipping. Presently, these options are seldom used, because they do not allow door-to-door delivery. In the future, the operation grade of carriers will increase significantly and the European Commission prognoses a suboptimal load of rail and waterways compared to the immoderate quantity of road transports [1].

The European road transport system is almost overloaded and currently does not offer enough potential for technological enhancement to face the future increase in traffic [2]. Hence, balancing the modes of transport i.e. intermodal transport is crucial for European transport policies.

Today's transport system has to face various challenges in

S. Jursch, S. Bischoff, E. Hauck, P. Flachskampf, K. Henning and S. Jeschke, IMA/ZLW \& IfU of RWTH Aachen University terms of safety, reducing traffic congestion and the improvement of loading processes and interoperability of available transport modes. For a competitive intermodality, the quality and flexibility of interfaces between modes and national transport chains needs to be increased.

Additionally, transport costs may be reduced by pursuing the trend towards high-volume loading units. Concerning the dimensions of the loading units, some transport modes may not meet the requirements of every cargo. The lack of standardization of intermodal loading units inhibits the connectivity of modes and generates costs e.g. through the requirement of special transshipment technologies.

Especially developed transport solutions, like the high-cube containers, jumbo semitrailers, curtain-side swap-bodies or boxes with liftable tops, were introduced to the market. However, those specialized solutions often can only be used for some applications and require special operational technologies so that extra costs arise.

The cost efficient use of intermodal loading units depends on several factors like time for loading and unloading, range of interoperability, theft protection, cargo area, loading height etc.

Despite the positive development of the modal split, intermodal transport is hampered by technical constraints particularly when it comes to the standardization of the loading units and transshipment technologies. Therefore intermodal transport lacks all-purpose products.

The project "TelliBox - Intelligent MegaSwapBoxes for advanced intermodal freight transport" has been launched by the European Commission due to the increasing demands from politics and society to counteract the trend towards increasing freight transport on roads. It focused on the development of an all-purpose loading unit, applicable in intermodal transport of road, rail, inland and short sea shipping for the use in Europe and Russia. The aim is to optimize the concept and design of swap-bodies by combining the advantages of containers and semitrailers for a sustainable intermodal freight transport. The objectives of this project are based on the experience of logistic companies, who have been operating in intermodal freight transport for years.

Such a loading unit needs to face several requirements [3] regarding its design and functionality (Fig. 1). It has to be trimodal, be stackable and applicable for handling from the top, use existing low floor wagons for rail transport, have an optimized cargo volume of $100 \mathrm{~m}^{3}$ with an internal height of $3 \mathrm{~m}$ and length of 45 feet, have loading facilities from three sides (completely openable doors), offer improved safety features against pilferage. 


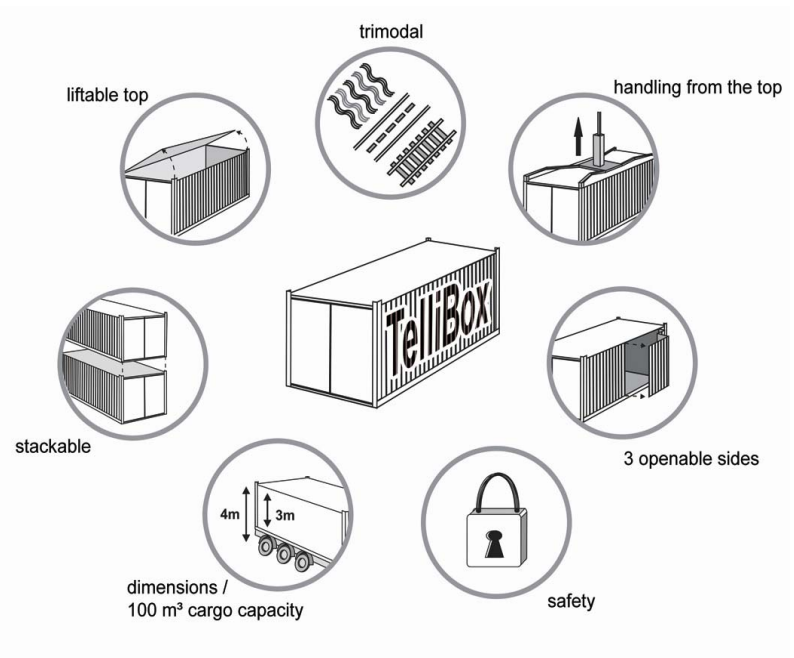

Figure 1. Requirements the ILU has to fulfill

\section{B. Problem Definition}

For the evaluation of the designed intermodal loading unit, a bundle of influencing criteria, effects and interactions have to be taken into consideration. Hence, for the analysis and evaluation of the profitability of a loading unit a holistic approach must be applied, which takes any effect into account. Traditional profitability analysis cannot - or just with great difficulty - include these influences and consequences into their approaches. The Value Oriented Cost-Effectiveness Estimation was developed in 2000 by Weydandt [276] to combine the classical analysis of investment in monetary terms with relevant non-monetary variables. With NOWS, the direct cost and benefit factors of several investment of specific scenarios or objects of investigation can be measured. For the designed loading unit, a potential market launch could only be successful, if the benefits of all effects are bigger than its costs. This will be evaluated by the later introduced method in terms of its profitability. This estimation should support the decision for a possible future investment.. The view on the solution contains the profitability of freight forwarding companies that use the ILU for their transports. This view focuses on the profitability of the new loading unit on a microeconomic level. The key emphasis here is whether or not the ILU would be profitable for a freight forwarding company. Therefore this paper focuses on the described perspective in order to answer that question. Hence, within the next chapter the design of the new intermodal loading unit is described. The Value Oriented Cost-Effectiveness Estimation will be introduced afterwards.

\section{INTERMODAL LOADING UNIT FOR ADVANCED INTERMODAL FREIGHT TRANSPORT}

\section{A. Designs for the MegaSwapBox}

The project TelliBox has to look for new design concept of ILUs to fulfill the above stated requirements. Different design workshops were held to discuss and find new designs. In these workshops the following solution proposal was designed, further developed and built up as a prototype.

\section{B. Intermodal loading unit design}

The main idea for the ILU is based on a shoe carton. A closed shoe carton is more stable, but if one removes one or more sides of the shoe carton, and then it becomes unstable. Using this construction principle, it stabilises the ILU if the side doors are part of the body construction (Fig. 2). It is therefore important for this construction, to design the door hinges and door closing elements so that they can take part of the forces which react on the ILU when it is being handled. The bottom frame is made up from a simple steel welded construction. To reduce the weight and have a stable construction, the bottom frame cross members have a trapez form. The lop layer of the bottom frame has a steel sheet as the loading platform. The bottom frame itself has an integrated goose neck tunnel. It is used for loading the container on the chassis so that the total height of chassis and container does not exceed the maximum height of $4 \mathrm{~m}$. The front walls are conventional container front walls which are currently used in the container construction The side doors are made up of 8 single doors and are designed flat.

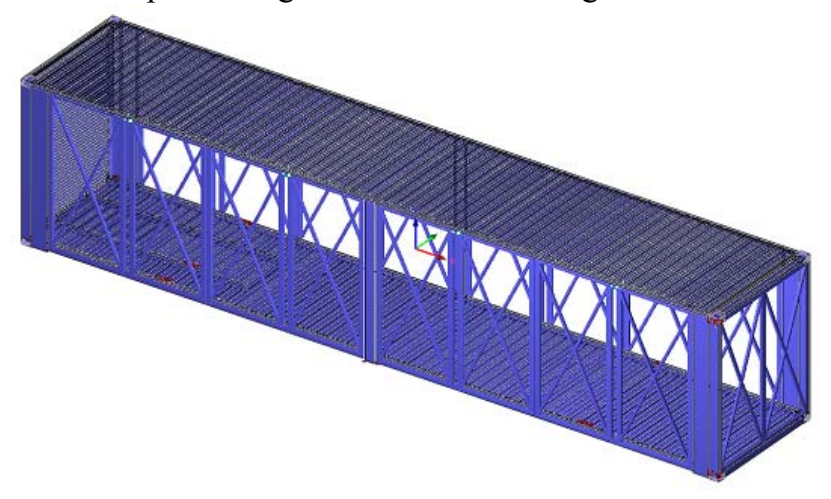

Figure 2. ILU solution

\section{INTRODUCTION TO THE VALUE ORIENTED COST-EFFECTIVENESS ESTIMATION}

It is always difficult to ascertain the benefits and costs of new innovative technologies. However, the economic evaluation of the respective costs and benefits is especially important during the planning and development process.

The method of the Value Oriented Cost-Effectiveness Estimation, also named as NOWS method [4], [5], [6], [7], developed by Weydandt in 2000 [276], is a participation-oriented, entrepreneurial cybernetic approach for the evaluation of costs and benefits of an investment. The NOWS method is a participation-oriented, entrepreneurial cybernetic approach for the evaluation of costs and benefits of an investment. The method enables a decision maker on investment opportunities to combine the classical analysis of investment in monetary terms with relevant non-monetary variables or the so-called soft factors in the profitability analysis. The method consists of a monetary examination and an integrated examination of the costs and benefits and it takes into consideration the currently on the market available products. The NOWS method combines the classical analysis of investment in monetary terms with relevant non-monetary variables or the so-called soft factors in the profitability analysis. This approach needs to be considered in comparison 
to the classical economic efficiency approaches: these conventional approaches limit themselves basically to the quantifiable objectives in terms of monetary data, e. g. costs and revenues (so called "hard" factors). In contrast, the NOWS approach considers non-monetary objectives such as time, quality, flexibility, employee perspectives or enterprise environment to be evaluated in terms of money. Therefore, with NOWS, the different factors for evaluation are distinguished as "direct, indirect and difficult to ascertain". The probabilities of occurrence of certain corresponding events are classified as "high, medium or low". Finally, the NOWS chart shows the monetized cost and benefit curves providing a profitability indicator for the investment. Therefore, this approach supplies the answer to the enterprise requirements which are partly taken from the guidelines for ISO-Certification 9000/2000.

The NOWS method consists of seven phases:

- Constitution of the Interdisciplinary Investment Team,

- Current-Situation-Analysis

- Target-Situation-Analysis

- Compilation of Measures

- Investment calculation and evaluation

- Implementation

- Reflection

They form the basis for all participation-oriented processes as well as the learning processes [8] of all participants and also the organization (Fig. 3). In the phase "Constitution of the Interdisciplinary Investment Team", a representative team is formed from executives and employees who are affected by the measures and strategies. This team conducts the whole evaluation, implementation and reflection process. During the phase "Current-Situation-Analysis", the existing skills and competencies in the enterprise are identified; in the "Target-Situation-Analysis" phase, the necessary competencies are derived from the strategic objectives, and are identified and classified according to the necessity of reaching them. Phase 4 "Compilation of Measures" defines individual strategies, which are then tested for their economic efficiency in the "Investment calculation and evaluation" phase. This is the core phase of the NOWS method. Within this phase, the costs and benefits of the investment are defined and assigned if they are direct, indirect or difficult to ascertain. They are also assigned regarding their probabilities of occurrence(high, medium or low). The evaluation is concluded by the decision to implement one or several strategies. The benefits as well as the costs get summarized in a $3 \times 3$-matrix containing the occurrence probability and the ascertain ability. Afterwards costs and benefits are monetized and accumulated from direct ascertain ability and high probability of occurrence to difficult ascertain ability and low probability of occurrence. Afterwards the accumulated costs and benefits get displayed in a NOWS chart. The intersection point of the cost graph and the benefit graph shows if the investment should be accomplished or refused. It is recommended in the "Implementation" phase, that participants from the Interdisciplinary Investment Team directly accompany the process, so that the advice for the evaluation of strategies is directly incorporated into the transfer process. In the "Reflection" phase, the Interdisciplinary Investment Team appraises the experiences gathered during the process (planning, evaluation, implementation and supervision), and it issues recommendations on possible actions for the next implementations, or for the realization of subsequent training and further education processes.

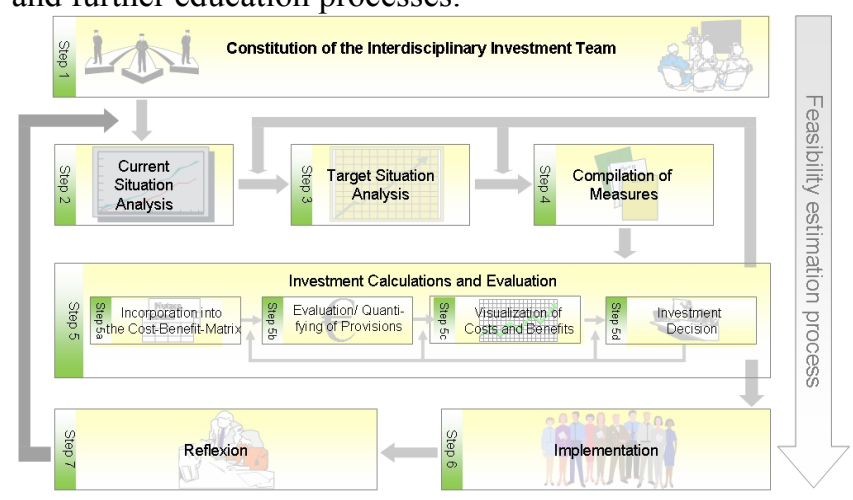

Figure 3. Value Oriented Cost-Effectiveness Estimation, the NOWS Method [7].

IV. Application of the VAlue ORIENTED COST-EFFECTIVENESS ESTIMATION ON INTELLIGENT MEGASWAPBOXES FOR ADVANCED INTERMODAL FREIGHT TRANSPORT

\section{A. Framework}

The Current-Situation-Analysis shows that the openings of boarders in the East of the European Union result in an increase of freight transport volume. The European Union's objectives of achieving intermodal integration and operational optimization can therefore be regarded as the definition of the "Target-Situation-Analysis" (phase three). Intelligent MSBs for Advanced Intermodal Freight Transport can be seen as one possible action to achieve this target situation by shifting the intermodal traffic in favor of rail and short sea traffic (phase four "Compilation of Measures").

The estimation of the costs and benefits had to be done by an Interdisciplinary Investment Team for the intermodal loading unit. It was agreed by the consortia of the project that for the design all annually costs and benefits for a freight forwarder operating with the ILU had to be evaluated in 2015. At this time it was assumed that the ramp-up phase would have been completed. To achieve an independency, the Investment calculation and evaluation are carried out relatively to standardized swap-bodies which are used today. All benefits respectively costs were classified in their type as well as their probability of occurrence.

The group consisted of designated experts. On the basis of a matrix all aspects were quantified by all participants together.

\section{B. Benefit Aspects}

In the following, the derivation of several selected benefit and cost aspects is described.

As a main direct benefit with high occurrence, a reduction of transport costs was identified by the participants of the workshop. Referring to a test track (Gliwice/Poland - 
Ellesmere Port/England) (Fig. 4), that was performed with the ILU, it was agreed that an intermodal transport with the new ILU has an advantage comparing it with a Megatrailer or a mega swap body on road, regarding the costs of $1,40 €$ per $\mathrm{km}$. Calculating the test track one way with $1,650 \mathrm{~km}$ and 24 cycles within one year and additionally assuming the costs of $341 €$ for the ferry and $117 €$ for the toll charge, expenses of approximately $2.768 €$ occur. Using the ILU as an intermodal transport especially by rail, this costs can be reduced by an amount of $660 € /$ cycle, which results in an annual saving of $16.224 €$ per year and box regarding 24 cycles.

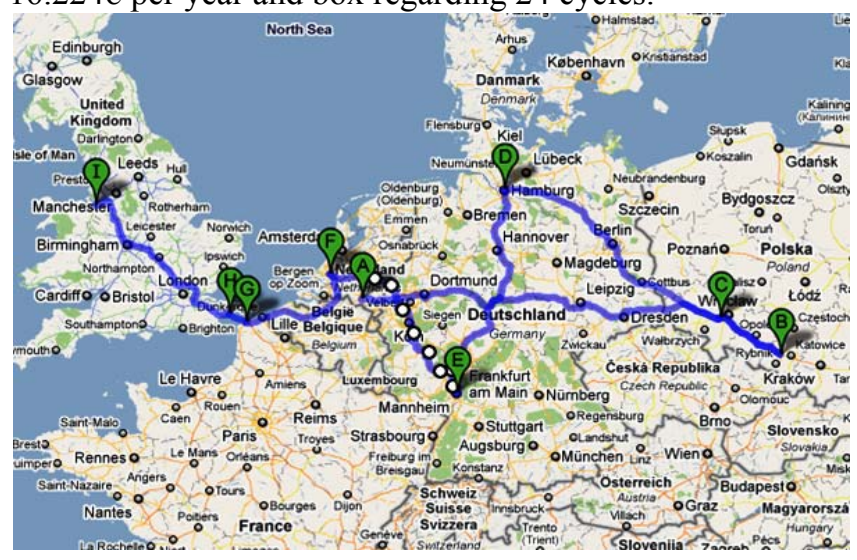

Figure 4. Test track for the intermodal loading unit

Since Megatrailers are not used in Eastern Europe, a comparison between the ILU and the Megatrailer seemed inappropriate on this test track. Therefore, the workshop members assumed a second test track from Germany to Portugal $(2000 \mathrm{~km})$. Constructed to be applied on railway as well, once again the monetary benefit of the ILU is underlined: While the trailer costs $3.000 €$ one way, the ILU is assumed to be approximately $10 \%$ cheaper $(2.700 €)$, which is a gain of $300 €$ one way, and $600 €$ a cycle. Considering 20 cycles on this section this results in an overall benefit of $12.000 €$ per year and box.

Another benefit is the special height of 3 meters of the ILU. Compared to the Megatrailer it is 3 centimeters higher and therefore a lucrative and highly demanded transporting tool for the automotive industry. Due to this 3 meters loading height, forwarders are in the position to gain exclusive transport orders - e.g. car body transports, which need more transporting space than the standard of $2,97 \mathrm{~m}$ offered by a Megatrailer. However, the monetary benefit of this special loading height is difficult to ascertain. Therefore, the workshop members conceived a possible scenario of an exclusive transport order with an assumed turnover of 10 Mio€ per year and a profit before taxes of $2 \%$, which leads to a benefit of $200.000 €$ per year. With the use of e.g. 300 ILUs, the forwarder would gain a net benefit of $660 €$ per box each year.

One further identified advantage of the ILU is its high protection against theft. The ILU can be transported to Eastern Europe without any reservation due to its solid construction. The Megatrailer, however, is not used in Eastern Europe at all, since its theft protection is marginal and completely insufficient. Therefore, in this respect, a comparison is inappropriate and monetary benefit difficult to ascertain. Nevertheless it should be mentioned, that with about 1.500 Megatrailers the annual financial loss amounts to around $150.000 €$ due to a low theft-protection. So the forwarders have to pay about $100 €$ per trailer every year, while the ILU's loss ranges from $0-5 €$ per box and year.

This high protection against theft allows a smooth transportation to Eastern Europe, which is a main destination for the ILU. By comparison, the Megatrailer depends on expensive security staff. Assuming a transportation via 4 trucks, each one will need 4 security people (two in the front, two in the back) to protect them. Assuming further, the staff costs $500 €$ a day and the service lasts five days, personnel costs of $2.500 €$ arise (500€ x 5days), which makes a total cost of $625 €$ per truck $(2.500 € / 4$ trucks $)$ and a total amount of $15.000 €$ a year, assuming 24 circulations $(24 \times 625 €=$ $15.000 €)$. This money can be saved by using the ILU.

The costs of minor damages during crane handling, whereas, are difficult to ascertain. Considering the test track from Gliwice to Ellesmere Port, the workshop-members estimated four crane manoeuvres per cycle ( 2 for the load and 2 for the unload) The loading is assumed to be the critical step, while there are no damages expected on unloading. Assuming every 10 th loading causing a damage with a value of $250 € 24$ circulations per year result in 48 loadings. This lead to roundabout 5 damages per year adding up to annual damage costs of $1250 €(250 €$ x $5=1250 €)$. These costs can be avoided by the use of the ILU which is resistant to such damages.

Furthermore, general maintenance costs like the sealing of the roof, replacing of hinges, tyres and brakes arise on a yearly basis. Due to experience value, those costs amount to $10 \%$ of the acquisition costs, namely $2.800 €$ regarding the ILU with an acquisition value of $28.000 €$. Additionally, $2.500 €$ need to be calculated for the replacement of the tyres and brakes, since they have to be exchanged every second year and cost $5.000 €(5.000 € / 2$ years $=2.500 € /$ year $)$. As the ILU uses just a $1 / 5$ of the chassis, only $1 / 5$ part of the value will be implemented into account $(2.500 € / 5=500 €)$. That makes a total cost of $3.300 €$ per year $(2.800 €+500 €)$. Using the Megatrailer, however, the forwarder has to spend $4.900 €$ a year $(4.000 €$ tyres and brakes $+900 €$ tarpaulin and planks) for the maintenance. This is $1.600 €$ more every year than he would spend using the ILU (4.900€-3.300€).

Moreover, a Megatrailer needs 13 lashing straps à $20 €$ to secure the goods. These straps have to be replaced every year. This causes an extra expense of $260 €+35 \%$ abrasion and amounts to $351 €$ a year. These costs can be avoided using the ILU, since it does not need any lashing straps due to its special construction.

The storage costs, whereas, affect both containers the same way, since both need to be stored temporarily and the storage fee remains the same. Even if there will be different pricing in the future (reduced costs for stackable ILU) the expected benefits for the ILU are marginal. The effective timesaving during the crane handling is not monetized, since the process of the handling endures just about half an hour and is therefore too marginal to be considered here. Furthermore, the image gain due to the $\mathrm{CO} 2$ savings for example, is one of the strongest arguments for the ILU, but very difficult to ascertain and therefore not monetized as well. 


\section{Cost Aspects}

As one main cost aspect, acquisition costs amounting to $32.000 €$ were identified by the workshop-participants. These costs include $28.000 €$ for the ILU and $1 / 5$ of the chassis-costs, which is $4.000 €$, since the ILU uses just $1 / 5$ of the chassis. (It is expected, that a forwarder will need on an average one chassis per five ILUs). The workshop-members agreed that for the ILU an amortization period of five years is realistic. Given an interest rate of $6 \%$, an amortization rate of $8.320 €$ per year can be calculated for the ILU over five years, which is $22,78 €$ per day. In contrast, the Megatrailer costs $25.000 €$. Applying the same conditions, the Megatrailer costs just $6.500 €$ per year and $17,80 €$ per day. Thus, the ILU is $1.820 €$ more expensive than the Megatrailer per year and box.

Above this, repair costs of the side doors are assumed to arise on a yearly basis for the ILU. These costs were classified as direct costs with a medium probability of occurrence. Calculating 24 cycles per year, a damage of the side doors with every sixth cycle is assumed. This means that four damages per year need to be taken into consideration. Further assuming $1.500 €$ worth of each damage makes a total of $6.000 €$ repair costs every year per box.

With its 24t loading capacity, the Megatrailer is able to load 2,5t more freight per box than the ILU with its $21,5 \mathrm{t}$. This leads to less annual revenues for the ILU considering the test track from Gliwice to Ellesmere Port and regarding just the rail way of $1.300 \mathrm{~km}$ and the costs of $1,40 € / \mathrm{km}$. To estimate a financial loss, the participants of the workshop had to determine the costs of one ton of freight. Calculating $1.300 \mathrm{~km} \times 1,40 € / \mathrm{km}$ a revenue of $1.820 €$ per box can be achieved. Divided by 24 tons this results in $75,83 €$ profit per ton. With its 2,5 tons less loading capacity the ILU has a monetary loss of $190 €$ per box. Furthermore, estimating 24 cycles, we have to assume a total annual loss of $4560 €$ per year and box.

Since the forwarder is faced with fixed costs per rail-trip each way and normally has a contract for one-way delivery, the workshop members decided to take empty trips on the return path into consideration, as well. The forwarder has to find a costumer who needs to deliver goods on the return path. A trip one way by train costs $1.080 €$ per box, calculating $60 \%$ of the possible revenue of $1.800 €$. Three scenarios with different probabilities have been developed during the workshop:

1) With high probability will there be additional costs (compared to the megatrailer) due to the reduced loading capacity, even if $100 \%$ of the return path are fully loaded. This are additional $4.560 €$ per year and box.

2) With medium probability there will be $40 \%$ percent of empty return path trips. Regarding 24 cycles per year, this means about 14 loaded trips and 10 empty trips. That leads to the following additional costs: Due to the smaller loading capacity of the ILU, calculated $190 €$ loss per box x 14 trips were calculated, which amounts to $2.660 €$ loss per box and year. In addition to the sum of $10.800 €$ per box and year, due to the remaining 10 empty trips $(10 \times 1.080 €)$, this adds up to a total amount of $13.460 €$ loss per year and box.

3) With low probability, $100 \%$ of the return path trips will be empty, because the forwarder cannot find a costumer for 21,5t transports. This would mean a total loss of $25.920 €$ per year and box (24 trips with train will cost $1.080 €)$.

For a correct calculation with the NOWS-tool, only the additional costs comparing the three scenarios can be taken into account. The additional costs for scenario b) are the total costs for scenario b) minus the costs for scenario a): $13.460 €$ - $4560 €=8900 €$. The additional costs for scenario c) are the total costs for scenario c) minus the total costs of scenario $b$ ): $25.920 €-13.460 €=12460 €$.

\section{Results of the Value Oriented Cost-Effectiveness Estimation}

Fig. 5 illustrates the resulting NOWS-graph for the Intermodal loading unit. The graph shows an intersection point between sector 4 and 5. This means that an investment in a new loading unit can be considered with a slightly positive trend.

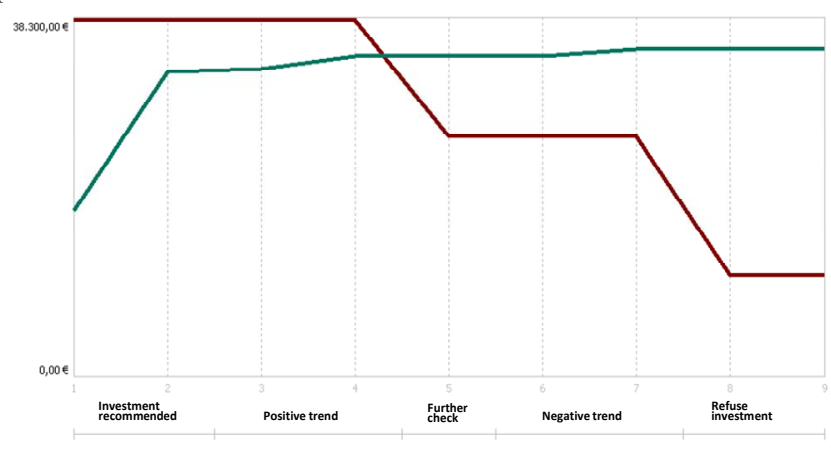

Figure 5. NOWS-graphs of the ILU

After evaluating the results of the NOWS-method it can be stated that an investment for an intermodal loading unit with the currently estimated price within the stage of prototyping can still be recommended.

\section{CONCLUSION}

Within this investigation, an estimation of the value orientated cost-effectiveness was conducted for a new intermodal loading unit. Participants of the workshop were members of the project consortia of TelliBox. The cost-effectiveness was evaluated relative to today used loading units.

Generally it could be shown that the benefits for the ILU slightly dominate the costs independent of the risk attitude of the decision maker.

The forwarders view and needs can be seen in the different monetized aspects. Nevertheless, the problem arose during the workshop, that the aspects are not easily comparable, especially because they are based on different scenarios and do not cover only one test track scenario. On the other hand the limitation to one test track scenario (e.g. the above mentioned Gliwice/Poland - Ellesmere Port/England) would have led to consider only a few from the possible cost and benefit aspects from ILU. It was decided during the workshop not to estimate only one scenario, but to take as many as possible aspects into account.

Therefore the results cannot be considered as one realistic scenario, but as a mixed set of possible aspects, which are 
critical for the success of the intermodal loading unit and need to be taken into account when planning a series production. According to that the NOWS graph does not show one evaluation of investment-decision for freight forwarders. Actually, the investment decisions which will be made will base on actual fright contracts and detailed data.

\section{ACKNOWLEDGMENT}

The research leading to these results has received funding from the European Community's Seventh Framework Programme (FP7/2007-2013) under grant agreement $n^{\circ} 217856$.

\section{REFERENCES}

[1] European Commission. Midterm-review of the White Paper on European transport policy, European Commission (EC), Brussels. 2006.

[2] Commission of the European Communities. Keep Europe moving -Sustainable mobility for our continent. Brussels, 2006.

[3] TelliBox Deliverable no. D2. Report requirement specification, www.TelliBox.eu. 2008.

[4] G. Strina, and J. Uribe. Participative pre-evaluation of the profitability of operational investments - NOWS approach - A process and involvement oriented evaluation method. In: Applied Participation and Empowerment at Work - Methods, Tools and Case Studies. Lund, 2004, pp. 81-92.

[5] Weydandt, Dirk: Beteiligungsorientierte wirtschaftliche Bewertung von technischen Investitionen für prozessorientierte Fertigungsinseln. Unternehmenskybernetik in der Praxis, Band 2. Aachen: Shaker-Verlag, 2000

[6] G. Strina, J. Uribe, K. Henning. NOWS Technique: Participation oriented Evaluation of further Education Strategies and Measures. In: Proceedings of E-Learn 2003. World Conference on E-learning in Corporate, Government, Healthcare \& Higher Education, Association for the Advancement of Computing in Education (AACE). Phoenix (USA), 2003, pp. $331 \mathrm{ff}$.

[7] J. Uribe, K. Henning, G. Strina. Measurement of Participation-oriented Evaluation: NOWS. In: Knowledge is Orange - Industry-Focused Applications of Knowledge Management across Germany. 2004.

[8] H. Unger. Organisationales Lernen durch Teams - Methode und Umsetzung eines teambasierten Projektsmanagements. München, and Mering, 1998. 